\title{
An Interval of No-Arbitrage Prices in Financial Markets with Volatility Uncertainty
}

\author{
Hanlei Hu, Zheng Yin, and Weipeng Yuan \\ School of Economic and Mathematics, Southwestern University of Finance and Economics, Chengdu, Sichuan 611130, China \\ Correspondence should be addressed to Weipeng Yuan; yuan_weipeng@sina.com
}

Received 21 February 2017; Accepted 11 May 2017; Published 19 June 2017

Academic Editor: Honglei Xu

Copyright (C) 2017 Hanlei Hu et al. This is an open access article distributed under the Creative Commons Attribution License, which permits unrestricted use, distribution, and reproduction in any medium, provided the original work is properly cited.

\begin{abstract}
In financial markets with volatility uncertainty, we assume that their risks are caused by uncertain volatilities and their assets are effectively allocated in the risk-free asset and a risky stock, whose price process is supposed to follow a geometric $G$-Brownian motion rather than a classical Brownian motion. The concept of arbitrage is used to deal with this complex situation and we consider stock price dynamics with no-arbitrage opportunities. For general European contingent claims, we deduce the interval of no-arbitrage price and the clear results are derived in the Markovian case.
\end{abstract}

\section{Introduction}

Though many choice situations show uncertainty, owing to the Ellsberg Parasox, the impacts of ambiguity aversion on economic decisions are established and Beissner [1] considered general equilibrium economies with a primitive uncertainty model that features ambiguity about continuoustime volatility. Under uncertainty, multiple priors can be used to model decisions. Recently, these multiple priors models have attracted much attention. The decision theoretical setting of multiple priors was introduced by Gilboa and Schmeidler [2] and Artzner et al. [3] adapted it to monetary risk measures. Afterwards, Maccheroni et al. [4] generalized multiple priors to preferences. In diffusion models, Girsanov's theorem was employed to consider stochastic processes by Chen and Epstein [5], but these multiple priors can only lead to uncertainty. When these multiple priors are used in finance areas, they result in drift uncertainty for stock prices. In the risk-neutral world, when we assess financial claims, the uncertainty of this drift will disappear.

Under the assumption of no arbitrage and volatility uncertainty, Fernholz and Karatzas [6] considered to outperform the market. Compared with this, our paper is to model volatility uncertain financial markets which have no arbitrage. Epstein and Ji [7] or Vorbrink [8] used a specific example to illustrate an uncertain volatility model. On the basis of our predecessors, our paper solves a few basic problems of the volatility uncertainty in finance markets. Our aim is to analyze the volatility uncertain financial markets and we take advantage of the framework of sublinear expectation and $G$-Brownian motion which is introduced by Peng [9] to deal with the model in financial markets. The $G$-Brownian motion is no longer a classical Brownian motion. The construction of stochastic integration, Itôs lemma, and martingale theory is utilized to the framework of $G$-Brownian motion. In order to control the model risk, the $G$-Brownian motion is employed to concern the model and evaluate claims by means of $G$ expectation which is a sublinear expectation.

In our financial markets with volatility uncertainty, the wealth is invested in risk-free asset and risky asset, in which the risky asset, that is, stock $S_{t}$ and its price process $S_{t}$, is given by the following geometric $G$-Brownian motion:

$$
d S_{t}=r S_{t} d t+v_{t} S_{t} d B_{t}, \quad S_{0}=x_{0}>0,
$$

where constant interest rate $r \geqslant 0$ is an expected instantaneous return of the stock and $v_{t}$ is the volatility of $S$ which is associated with $t$. The canonical process $B=\left(B_{t}\right)$ is a $G$-Brownian motion relating to a sublinear expectation $E_{G}$, called $G$-expectation (see $[9,10]$ for a detailed construction). The stochastic calculus with respect to G-Brownian motion can also be established, especially Itô integral [9]. The 
ordinary martingales are replaced by $G$-martingales. Denis et al. [11] developed the G-framework of Peng [10] (see [12]) in the framework of quasi-sure analysis. An upper expectation of classical expectations is used to represent the sublinear expectation $E_{G}$ established by Denis et al. [11]; that is to say, there exists a set of probability measures $\mathbf{P}$ such that $E_{G}[X]=$ $\sup _{P \in \mathbf{P}} \mathbb{E}^{P}[X]$.

In this paper, we prove that the considered financial market does not admit any arbitrage opportunity, but it allows for uncertain volatility. In our analysis, the notion of $G$-martingale which replaces the notion of martingale in classical probability theory plays a major role.

One of our aims is to solve

$$
\begin{aligned}
& \sup _{P \in \mathbf{P}} \mathbb{E}^{P}\left(D_{T} V_{T}\right), \\
& \sup _{P \in \mathbf{P}} \mathbb{E}^{P}\left(-D_{T} V_{T}\right),
\end{aligned}
$$

where $V_{T}$ denotes the payoff of contingent claims at maturity $T$ and $D_{T}$ is a discounting. $\mathbf{P}$ presents a series of different probability measures.

The stochastic environment can bring about a set of probability measures that are not equivalent but even mutually singular. To illustrate this, let $B$ be a Brownian motion under a measure $P$ and think about the processes $S^{\underline{\sigma}}:=\left(\underline{\sigma} B_{t}\right)$ and $S^{\bar{\sigma}}:=\left(\bar{\sigma} B_{t}\right)$. Using $P^{\underline{\sigma}}=P \circ\left(S^{\underline{\sigma}}\right)^{-1}$ and $P^{\bar{\sigma}}=P \circ\left(S^{\bar{\sigma}}\right)^{-1}$, we describe the distributions over continuous trajectories which are induced by the two processes. These measures describe two possible hypotheses of real probability measure which drives the volatility uncertainty by (1). Therefore, we have

$$
P^{\underline{\sigma}}\left(\left\{\langle B\rangle_{T}=\underline{\sigma}^{2} T\right\}\right)=1=P^{\bar{\sigma}}\left(\left\{\langle B\rangle_{T}=\bar{\sigma}^{2} T\right\}\right),
$$

where both priors are mutually singular.

The definition of trading strategy and portfolio process is applied to obtain the wealth equation. Defining the concept of no-arbitrage in financial markets and the hedging classes, we gain the interval of no-arbitrage price for general European contingent claims. Finally, the connection of the lower and upper arbitrage prices is presented.

In such an ambiguous financial market, our subject is to analyze the European contingent claim concerning pricing and hedging. The asset pricing is extended to the financial markets with volatility uncertainty. The notion of no-arbitrage plays an important role in our analysis. Owing to the fact that the volatility uncertainty leads to additional source of risk, the classical definition of arbitrage will no longer be adequate. For this reason, a new arbitrage definition is presented to adjust our multiple priors model with mutually singular priors which are shown in (3). In this modified sense, we confirm that our volatility uncertain financial markets do not admit any arbitrage opportunity.

Utilizing the notion of no-arbitrage, we have obtained several results, which provide us with a better economic understanding of financial markets under volatility uncertainty. For general contingent claims, we determine an interval of no-arbitrage prices. The bounds of this interval are the upper and lower arbitrage prices $v_{\text {up }}$ and $v_{\text {low }}$, which are obtained as the expected value of the claim's discounted payoff with respect to $G$-expectation (see (2)). They specify the lowest initial capital. We use the capital to hedge a short position in the claim or long position, respectively. Generally speaking, because $E_{G}$ is a sublinear expectation, we have $v_{\text {low }} \neq v_{\text {up }}$. This verifies the market's incompleteness. In a few words, no arbitrage will be generated when price is in the interval $\left(v_{\text {low }}, v_{\text {up }}\right)$ for a European contingent claim. In Section 4, when the contingent claim's payoff is only determined by the current stock price, we deduce a more clear structure about the upper and lower arbitrage prices by a partial differential equation (PDE for short). We derive an explicit representation for the corresponding supper-hedging strategies and consumption plans. Given the special situation when the payoff function shows convexity (concavity), the upper arbitrage price solves the classical Black-Scholes PDE with a volatility equal to $\underline{\sigma}(\bar{\sigma})$, and vice versa concerning the lower arbitrage price.

The novelties of this paper are that the volatility of $S$ in our model is a variable which is related to $t$. This is different from works of Vorbrink [8] in which the volatility of $S$ is a constant. We employ the $G$-framework including $G$-expectation, $G$ Brownian motion, and the concept of arbitrage to study the financial markets with volatility uncertainty; we gain the interval of no arbitrage, which is different from that in Denis and Martini [12].

This paper is organized as follows. Section 2 introduces the financial markets. We focus on and extend the terminology from mathematical finance. Section 3 applies a series of definitions and lemmas to derive the interval of no arbitrage. Section 4 restricts us to the Markovian case and derives results which are analogy to those in Avellaneda et al. [13] or Vorbrink [8]. Conclusions are given in Section 5.

\section{The Market Model and the Mathematical Setting}

2.1. G-Brownian Motion and the Multiple Priors Setting. In the whole paper, the one-dimensional case is considered and we fix an interval $[\underline{\sigma}, \bar{\sigma}]$ with $\underline{\sigma}>0$. This interval describes the volatility uncertainty. $\underline{\sigma}$ and $\bar{\sigma}$ denote a lower and upper bound for volatility, respectively.

Definition 1 (see [9]). Let $\Omega \neq \emptyset$ be a given set. Let $\mathscr{H}$ be a linear space of real valued functions defined on $\Omega$ with $c \in \mathscr{H}$ for all constants $c$, and $|X| \in \mathscr{H}$ if $X \in \mathscr{H}$. ( $\mathscr{H}$ is considered as the space of random variables.) A sublinear expectation $\widehat{E}$ on $\mathscr{H}$ is a functional $\widehat{E}: \mathscr{H} \rightarrow \mathbb{R}$ satisfying the following properties: for any $X, Y \in \mathscr{H}$, it has

(1) Monotonicity: if $X \geqslant Y$ then $\widehat{E}(X) \geqslant \widehat{E}(Y)$.

(2) Constant preserving: $\widehat{E}(c)=c$.

(3) Subadditivity: $\widehat{E}(X+Y) \leqslant \widehat{E}(X)+\widehat{E}(Y)$.

(4) Positive homogeneity: $\widehat{E}(\mu X)=\mu \widehat{E}(X) \forall \mu \geqslant 0$.

The triple $(\Omega, \mathscr{H}, \widehat{E})$ is called a sublinear expectation space. 
Definition 2 (see [10] (G-normal distribution)). In a sublinear expectation space $(\Omega, \mathscr{H}, \widehat{E})$, a random variable $X$ is called (centralized) $G$-normal distributed if for any $a, b \geqslant 0$

$$
a X+b \bar{X} \sim \sqrt{a^{2}+b^{2}} X,
$$

where $\bar{X}$ is an independent copy of $X$. Here the letter $G$ denotes the function

$$
G(a):=\frac{1}{2} \widehat{E}\left(a X^{2}\right): \mathbb{R} \longrightarrow \mathbb{R} .
$$

Note that $X$ has no mean-uncertainty; that is, it has $\widehat{E}(X)=\widehat{E}(-X)=0$. Moreover, the following important identity holds:

$$
G(a)=\frac{1}{2} \bar{\sigma}^{2} a^{+}-\frac{1}{2} \sigma^{2} a^{-}
$$

with $\sigma^{2}:=-\widehat{E}\left(-X^{2}\right)$ and $\bar{\sigma}^{2}:=\widehat{E}\left(X^{2}\right)$. We write that $X$ is $N\left(\{0\} \times\left[\sigma^{2}, \bar{\sigma}^{2}\right]\right)$ distributed. Therefore, we say that $G$-normal distribution is characterized by the parameters $0<\underline{\sigma} \leqslant \bar{\sigma}$.

Remark 3 (see [10]). The random variable $X$ which is defined in (4) is generated by the following parabolic PDE defined in $[0, T] \times \mathbb{R}$.

For any $C_{l, L i p}(\mathbb{R})$, define $u(t, x):=\widehat{E}[\varphi(x+\sqrt{t} X)]$; then $u$ is the unique (viscosity) solution of

$$
\partial_{t} u-G\left(\partial_{x x} u\right)=0,\left.\quad u\right|_{t=0}=\varphi .
$$

Equation (7) is called a $G$-equation.

Definition 4 (see [10] (G-Brownian motion)). A process $\left(B_{t}\right)_{t \geqslant 0}$ in a sublinear expectation space $(\Omega, \mathscr{H}, \widehat{E})$ is called a $G$-Brownian motion if the following properties are satisfied:

(i) $B_{0}=0$.

(ii) For each $t, s \geqslant 0$ the increment $B_{t+s}-B_{t}$ is $N\left(\{0\} \times\left[\underline{\sigma}^{2} s, \bar{\sigma}^{2} s\right]\right)$ distributed and independent from $\left(B_{t_{1}}, B_{t_{2}}, \ldots, B_{t_{n}}\right)$ for each $n \in \mathbb{N}, 0 \leqslant t_{1} \leqslant \cdots t_{n} \leqslant t$.

Condition (ii) can be replaced by the following three conditions giving a characterization of $G$-Brownian motion:

(i) For each $t, s \geqslant 0: B_{t+s}-B_{t} \sim B_{t}$ and $\widehat{E}\left(\left|B_{t}\right|^{3}\right) / t \rightarrow 0$ as $t \rightarrow 0$.

(ii) The increment $B_{t+s}-B_{t}$ is independent from $\left(B_{t_{1}}, B_{t_{2}}, \ldots, B_{t_{n}}\right)$ for each $n \in \mathbb{N}, 0 \leqslant t_{1} \leqslant \cdots t_{n} \leqslant t$.

(iii) $\widehat{E}\left(B_{t}\right)=-\widehat{E}\left(-B_{t}\right)=0, \forall t \geqslant 0$. motion.

For each $t_{0}>0$, it has that $\left(B_{t+t_{0}}-B_{t_{0}}\right)_{t \geqslant 0}$ is a $G$-Brownian

Let us briefly depict the construction of $G$-expectation and its corresponding G-Brownian motion. As in the previous sections, we fix a time horizon $T>0$ and set $\Omega_{T}=$ $C_{0}([0, T], \mathbb{R})$-the space of all real valued continuous paths starting at zero. Considering the canonical process $B_{t}(\omega)$ := $\omega_{t}, t \leqslant T, \omega \in \Omega$, we define

$$
\begin{aligned}
& L_{i p}\left(\Omega_{T}\right):=\left\{\varphi\left(B_{t_{1}}, \ldots, B_{t_{n}}\right) \mid n \in \mathbb{N}, t_{1}, \ldots, t_{n}\right. \\
& \left.\quad \in[0, T], \varphi \in C_{l, L i p}\left(\mathbb{R}^{n}\right)\right\} .
\end{aligned}
$$

A G-Brownian motion is firstly constructed in $L_{i p}\left(\Omega_{T}\right)$. For this purpose, let $\left(\xi_{i}\right)_{i \in \mathbb{N}}$ be a sequence of random variables in a sublinear expectation space $(\widetilde{\Omega}, \widetilde{\mathscr{H}}, \widetilde{E})$ such that $\xi_{i}$ is $G$ normal distributed and $\xi_{i+1}$ is independent of $\left(\xi_{1}, \ldots, \xi_{i}\right)$ for each integer $i \geqslant 1$. Then a sublinear expectation in $L_{i p}\left(\Omega_{T}\right)$ is constructed by the following procedure: for each $X \in$ $L_{i p}\left(\Omega_{T}\right)$ with $X=\varphi\left(B_{t_{1}}-B_{t_{0}}, B_{t_{2}}-B_{t_{1}}, \ldots, B_{t_{n}}-B_{t_{n-1}}\right)$ for some $\varphi \in C_{l, L i p}\left(\mathbb{R}^{n}\right), 0 \leqslant t_{0}<t_{1}<\cdots<t_{n} \leqslant T$, set

$$
\begin{aligned}
E_{G} & {\left[\varphi\left(B_{t_{1}}-B_{t_{0}}, B_{t_{2}}-B_{t_{1}}, \ldots, B_{t_{n}}-B_{t_{n-1}}\right)\right] } \\
:=\widetilde{E} & {\left[\varphi\left(\sqrt{t_{1}-t_{0}} \xi_{1}, \sqrt{t_{2}-t_{1}} \xi_{2}, \ldots, \sqrt{t_{n}-t_{n-1}} \xi_{n}\right)\right] . }
\end{aligned}
$$

The related conditional expectation of $X \in L_{i p}\left(\Omega_{T}\right)$ as above under $\Omega_{t_{i}}, i \in \mathbb{N}$, is defined by

$$
\begin{aligned}
& E_{G} {\left[\varphi\left(B_{t_{1}}-B_{t_{0}}, B_{t_{2}}-B_{t_{1}}, \ldots, B_{t_{n}}-B_{t_{n-1}}\right) \mid \Omega_{t_{i}}\right] } \\
&:=\psi\left(B_{t_{1}}-B_{t_{0}}, \ldots, B_{t_{i}}-B_{t_{i-1}}\right),
\end{aligned}
$$

where $\psi\left(x_{1}, \ldots, x_{i}\right):=\tilde{E}\left[\varphi\left(x_{1}, \ldots, x_{i}, \sqrt{t_{i+1}-t_{i}} \xi_{i+1}, \ldots\right.\right.$, $\left.\left.\sqrt{t_{n}-t_{n-1}} \xi_{n}\right)\right]$. One checks that $E_{G}$ consistently defines a sublinear expectation in $L_{i p}\left(\Omega_{T}\right)$ and the canonical process $B$ represents a $G$-Brownian motion.

Let $\Theta:=[\underline{\sigma}, \bar{\sigma}]$ and $\mathscr{A}_{0, T}^{\Theta}$ be the collection of all $\Theta$-valued $\left(\mathscr{F}_{t}\right)$-adapted processes on $[0, T]$. We write

$$
B_{t}^{0, \sigma}:=\int_{0}^{t} \sigma_{s} d B_{s}
$$

and $P^{\sigma}$ as the law of $B^{0, \sigma}=\int_{0}^{\cdot} \sigma_{s} d B_{s}$; that is, $P^{\sigma}=P_{0} \circ\left(B^{0, \sigma}\right)^{-1}$ is distribution over trajectories. Let the set of multiple priors $\mathbf{P}$ be the closure of $\left\{P^{\sigma} \mid \sigma \in \mathscr{A}_{0, T}^{\Theta}\right\}$ under the topology of weak convergence.

Theorem 5 (see [8]). For any $\varphi \in C_{l, L i p}\left(\mathbb{R}^{n}\right), n \in \mathbb{N}, 0 \leqslant t_{1} \leqslant$ $\cdots \leqslant t_{n} \leqslant T$, it holds that

$$
\begin{aligned}
E_{G} & {\left[\varphi\left(B_{t_{1}}, \ldots, B_{t_{n}}-B_{t_{n-1}}\right)\right] } \\
= & \sup _{\theta \in \mathscr{A}_{0, T}^{\Theta}} \mathbb{E}^{P}\left[\varphi\left(B_{t_{1}}^{0, \theta}, \ldots, B_{t_{n}}^{t_{n-1}, \theta}\right)\right] \\
= & \sup _{\theta \in \mathscr{A}_{0, T}^{\Theta}} E^{P^{\theta}}\left[\varphi\left(B_{t_{1}}, \ldots, B_{t_{n}}-B_{t_{n-1}}\right)\right] \\
= & \sup _{P^{\theta} \in \mathbf{P}} E^{P^{\theta}}\left[\varphi\left(B_{t_{1}}, \ldots, B_{t_{n}}-B_{t_{n-1}}\right)\right] .
\end{aligned}
$$

Furthermore,

$$
E_{G}(X)=\sup _{P^{\theta} \in \mathbf{P}} \mathbb{E}^{P}(X), \quad \forall X \in L_{G}^{1}\left(\Omega_{T}\right) .
$$

We use the set of priors $\mathbf{P}$ to define the $G$-expectation $E_{G}$. It is given by

$$
E_{G}(X)=\sup _{P \in \mathbf{P}} \mathbb{E}^{P}(X)
$$


where $X$ is any random variable. So the $G$-expectation can be defined. Relative to the $G$-expectation, the space of random variable is denoted by $L_{G}^{1}\left(\Omega_{T}\right)$.

In this paper, we consider the tuple as $\left(\Omega_{T}, \mathscr{F},\left(\mathscr{F}_{t}\right), \mathbf{P}\right)$ and the canonical process $B=\left(B_{t}\right)$ is a $G$-expectation motion with respect to $\mathbf{P}$ as given in the previous. The $G$-framework enables the analysis of stochastic processes for all priors of $\mathbf{P}$. The terminology of "quasi-surely" (q.s.) is proved to be very useful.

Unless there are special instructions, all equations should also be understood as "quasi-sure." This means a property almost surely for all conceivable scenarios.

As mentioned in the preceding, $G$-expectation can be defined in the space $L_{G}^{p}\left(\Omega_{T}\right), p \geqslant 1$. It is the completion of $\mathscr{C}_{b}\left(\Omega_{T}\right)$, the set of bounded continuous functions on $\Omega_{T}$ under the norm $\|\xi\|:=\left(E_{G}\left[|\xi|^{2}\right]\right)^{1 / 2}<\infty$. Because the stochastic integrals are required to define trading strategies in the next sections, we briefly introduce the basic concepts about stochastic calculus and the construction of Itô integral with respect to $G$-Brownian motion.

For $p \geqslant 1$, let $M_{G}^{p, 0}(0, T)$ be the collection of simple processes $\eta$ of the following form: for a given partition $[0, T]$, $\left\{t_{0}, t_{1}, \ldots, t_{N}\right\}, N \in \mathbb{N}$, for any $t \in[0, T]$ the process $\eta$ is defined by

$$
\eta_{t}(\omega):=\sum_{j=0}^{N-1} \xi_{i}(\omega) \mathbf{1}_{\left[t_{j}, t_{j+1}\right)}(t)
$$

where $\xi_{i}(\omega) \in L_{G}^{p}\left(\Omega_{t_{i}}\right), i=0,1, \ldots, N-1$. For each $\eta \in$ $M_{G}^{p, 0}(0, T)$, let

$$
\|\eta\|_{M_{G}^{p}}:=\left(E_{G} \int_{0}^{T}\left|\eta_{s}\right|^{p} d s\right)^{1 / p} .
$$

We denote by $M_{G}^{p, 0}(0, T)$ the completion of $M_{G}^{p, 0}(0, T)$ under the norm $\|\cdot\|_{M_{G}^{p} \text {. }}$

Definition 6 (see [14]). For $\eta \in M_{G}^{2,0}(0, T)$ with the presentation in (15), the integral mapping is defined by $I$ : $M_{G}^{2,0}(0, T) \rightarrow L_{G}^{2}\left(\Omega_{T}\right)$ and

$$
I(\eta)=\int_{0}^{T} \eta(s) d B_{s}:=\sum_{j=0}^{N-1} \xi_{i}\left(B_{t_{j+1}}-B_{t_{j}}\right) .
$$

We consider the quadratic variation process $\left(\langle B\rangle_{t}\right)$ of $G$ Brownian motion. It has

$$
\langle B\rangle_{t}=B_{t}^{2}-2 \int_{0}^{t} B_{s} d B_{s}, \quad \forall t \leqslant T .
$$

It is a continuous, increasing process, absolutely continuous with respect to $d t$. It contains all the statistical uncertainty of the $G$-Brownian motion. For $s, t \geqslant 0$ we have $\langle B\rangle_{s+t}-\langle B\rangle_{s} \sim$ $\langle B\rangle_{t}$ and it is independent of $\Omega_{s}$.
Definition 7 (see [9]). Let $x \in \mathbb{R}, z \in M_{G}^{2}(0, T)$ and $\eta \in$ $M_{G}^{1}(0, T)$. Then the process

$$
\begin{array}{r}
M_{t}:=x+\int_{0}^{t} z_{s} d B_{s}+\int_{0}^{t} \eta_{s} d\langle B\rangle_{s}-\int_{0}^{t} 2 G\left(\eta_{s}\right) d s, \\
t \leqslant T,
\end{array}
$$

is a $G$-martingale.

Specially, the nonsymmetric part $-K_{t}:=\int_{0}^{t} \eta_{s} d\langle B\rangle_{s}-$ $\int_{0}^{t} 2 G\left(\eta_{s}\right) d s, t \in[0, T]$, is a $G$-martingale, which is quite a surprising result because $\left(-K_{t}\right)$ is continuous, nonincreasing with quadratic variation equal to zero.

Remark 8 (see [15]). $M$ is a symmetric $G$-martingale if and only if $K \equiv 0$.

Theorem 9 (see [15] (martingale representation)). Let $\alpha \geqslant 0$ and $\xi \in L_{G}^{\alpha}\left(\Omega_{T}\right)$. Then the $G$-martingale $X$ with $X_{t}:=E_{G}(\xi \mid$ $\left.\mathscr{F}_{t}\right), t \in[0, T]$, has the following unique representation:

$$
X_{t}=X_{0}+\int_{0}^{t} z_{s} d B_{s}-K_{t}
$$

where $K$ is a continuous, increasing process with $K_{0}=0, K_{T} \in$ $L_{G}^{\beta}\left(\Omega_{T}\right), z \in H_{G}^{\beta}(0, T), \forall \beta \in[1, \alpha)$, and $-K$ a G-martingale.

If $\alpha=2$ and $\xi$ bounded from above, $z \in M_{G}^{2}(0, T)$ and $K_{T} \in L_{G}^{2}\left(\Omega_{T}\right)$ (see [14]).

A construction of the stochastic integral for the domain $H_{G}^{p}(0, T), p \geqslant 1$ is established by Song [15]. Although the structure of these spaces is similar as before, the norm for completion is different and the random variables $\xi_{i}(\omega)$ in (15) are elements of a subset of $L_{G}^{p}\left(\Omega_{t_{i}}\right)$. We will also use the domain $H_{G}^{1}(0, T)$ which is necessary for the martingale representation in the $G$-framework (see Theorem 9). For $p=2$, both domains coincide (see Song [15]). As a consequence, we can define the stochastic integral since $M_{G}^{2}(0, T)$ is contained in $H_{G}^{1}(0, T)$. In financial fields, more trading strategies will be feasible.

2.2. The Financial Market Model. We consider the following financial market $\mathscr{M}$ which includes a risk-free asset and a single risky asset and two assets are traded continuously over $[0, T]$. Assume that the risk-free asset is a bond and its interest rate is $r$. So the discount process $D_{t}$ can be defined to satisfy the following formula:

$$
d D_{t}=-r D_{t} d t, \quad D_{0}=1,
$$

where constant $r \geqslant 0$ is the interest rate of the riskless bond as in the classical theory.

Assume that the risky asset is a stock with price $S_{t}$ at time $t$, whose price process $S_{t}$ is given by the following equation:

$$
d S_{t}=r S_{t} d t+v_{t} S_{t} d B_{t}, \quad S_{0}=x_{0}>0,
$$

where $B=\left(B_{t}\right)$ denotes the canonical process which is a $G$-Brownian motion under $E_{G}$ or $\mathbf{P}$, respectively, with parameters $\bar{\sigma}>\underline{\sigma}>0$. 
Since $B=\left(B_{t}\right)$ is a $G$-Brownian motion, the volatility of $S$ is related to $t$ which is different from that of Vorbrink [8], where the volatility of stock price is a constant 1 . Consequently, the stock price evolution involves not only risk modeled by the noise part but also ambiguity about the risk due to the unknown deviation of the process $B$ from its mean. According to financial fields, this ambiguity is called volatility uncertainty.

Compared with the classical stock price process, (22) does not contain any volatility parameter $\sigma$. This is due to the characteristics of the G-Brownian motion $B$. Apparently, if we choose $\sigma=\underline{\sigma}=\bar{\sigma}$, then we will be in the classical BlackScholes model.

Remark 10. Take notice of the discounted stock price process $\left(D_{t} S_{t}\right)$ which is a symmetric $G$-martingale relative to the corresponding $G$-expectation $E_{G}$. As everyone knows, both the pricing and hedging of contingent claims are treated under a risk-neutral measure. This leads to a favorable situation in which the discounted stock price process is a (local) martingale [16]. In our ambiguous setting, this is also allowed. In order to model $\left(D_{t} S_{t}\right)$ as a symmetric $G$ martingale (see Definition 7), we do not need to change the sublinear expectation. A symmetric $G$-martingale is required to make sure that the stock is the same for all participants, whether they sell or buy.

Definition 11 (see [17]). In the market $\mathscr{M}$, a trading strategy is an $\left(\mathscr{F}_{t}\right)$-adapted vector process $(\alpha, \beta)=\left(\alpha_{t}, \beta_{t}\right), \beta$ a member of $H_{G}^{1}(0, T)$ such that $\left(\beta_{t} S_{t}\right) \in H_{G}^{1}(0, T)$, and $\alpha_{t} \in \mathbb{R}$ for all $t \leqslant T$.

A cumulative consumption process $\left\{C=\left(C_{t}\right), 0 \leqslant\right.$ $t \leqslant T\}$ is a nonnegative $\left(\mathscr{F}_{t}\right)$-adapted process with values in $L_{G}^{1}\left(\Omega_{T}\right)$, and with increasing, right-continuous, and $C_{0}=0$, $C_{T}<\infty$ q.s.

A basic assumption in the market $\mathscr{M}$ is that the stock price process $S_{t}$ defined by (22) is an element of $M_{G}^{2}(0, T)=$ $H_{G}^{2}(0, T)$. We impose the so-called self-financing condition. In other words, consumption and trading in $\mathscr{M}$ satisfy

$$
\begin{aligned}
& H_{t}:=D_{t}^{-1} \alpha_{t}+\beta_{t} S_{t} \\
&=\alpha_{0} D_{0}^{-1}+\beta_{0} S_{0}+\int_{0}^{t} \alpha_{u} d D_{u}^{-1}+\int_{0}^{t} \beta_{u} d S_{u}-C_{t}, \\
& \forall t \leqslant T \text { q.s., }
\end{aligned}
$$

where $H_{t}$ denotes the value of the trading strategy at time $t$. The meaning of (23) is that, starting with an initial amount $D_{0}^{-1} \alpha_{0}+\beta_{0} S_{0}$ of wealth, all changes in wealth are due to capital gains (appreciation of stocks and interest from the bond), minus the amount consumed. The q.s. means quasi-surely, which is the same as before.

For economic and mathematical considerations, it is more appropriate to introduce wealth and a portfolio process which presents the proportions of wealth invested in the risky stock.
Remark 12 (see [17]). A portfolio process $\pi$ represents proportions of a wealth $X$ which is invested in the stock. If we define

$$
\begin{aligned}
& \beta_{t}:=\frac{X_{t} \pi_{t}}{S_{t}}, \\
& \alpha_{t}:=X_{t} D_{t}\left(1-\pi_{t}\right),
\end{aligned}
$$

$$
\forall t \leqslant T,
$$

then we have $X_{t}=H_{t}$. As long as $\pi$ constitutes a portfolio process with corresponding wealth process $X$, the $(\alpha, \beta)$ is a trading strategy in the sense of (23).

Definition 13 (see [8]). A portfolio process is an $\left(\mathscr{F}_{t}\right)$-adapted real valued process if $\pi=\pi_{t}$ with values in $L_{G}^{1}\left(\Omega_{T}\right)$.

Definition 14. For a given initial capital $m$, a portfolio process $\pi$, and a cumulative consumption process $C$, consider the wealth equation

$$
\begin{aligned}
d X_{t} & =X_{t}\left(1-\pi_{t}\right) D_{t} d D_{t}^{-1}+X_{t} \pi_{t} \frac{d S_{t}}{S_{t}}-d C_{t} \\
& =X_{t} r d t+X_{t} \pi_{t} \nu_{t} d B_{t}-d C_{t}
\end{aligned}
$$

with initial wealth $X_{0}=m$. Or equivalently,

$$
D_{t} X_{t}=m-\int_{0}^{t} D_{u} d C_{u}+\int_{0}^{t} D_{u} X_{u} \pi_{u} v_{u} d B_{u}
$$

$$
\forall t \leqslant T \text {. }
$$

If this equation has a unique solution $X=\left(X_{t}\right):=X^{m, \pi, C}$, then it is called the wealth process corresponding to the triple $(m, \pi, C)$.

In the setup of Definition 14, notice that the $\int_{0}^{T} X_{t}^{2} \pi_{t}^{2} v_{t}^{2} d t<\infty$ must hold quasi-surely. Thus, we need to impose requirements $\left(\pi_{t} X_{t} v_{t}\right) \in H_{G}^{p}(0, T) p \geqslant 1$, or $\left(\pi_{t} X_{t} v_{t}\right) \in M_{G}^{p}(0, T), p \geqslant 2$.

Definition 15. A portfolio/consumption process pair $(\pi, C)$ is called admissible for an initial capital $m \in \mathbb{R}$ if

(i) the pair obeys the conditions of Definitions 11, 13, and 14 ,

(ii) $\left(\pi_{t} X_{t}^{m, \pi, C} v_{t}\right) \in H_{G}^{1}(0, T)$,

(iii) the solution $X_{t}^{m, \pi, C}$ satisfies

$$
X_{t}^{m, \pi, C} \geqslant-J, \quad \forall t \leqslant T, \text { q.s. }
$$

where $J$ is a nonnegative random variable in $L_{G}^{2}\left(\Omega_{T}\right)$.

We then have $(\pi, C) \in \mathscr{A}(m)$.

In the above Definitions 11 and $13-15$, it is necessary to guarantee that the financial fields and related stochastic analysis can be well defined. In particular, condition (ii) of Definition 15 makes sure that the mathematical framework does not collapse by allowing for many portfolio processes. 


\section{Arbitrage and Contingent Claims}

Definition 16 (see [8] (arbitrage in $\mathscr{M})$ ). We say that there is an arbitrage opportunity in $\mathscr{M}$ if there exist an initial wealth $m \leqslant 0$ and an admissible pair $(\pi, C) \in \mathscr{A}(m)$ with $C \equiv 0$ such that, at some time $T>0$,

$$
\begin{array}{cl}
X_{T}^{m, \pi, 0} \geqslant 0 & \text { q.s., } \\
P\left(X_{T}^{m, \pi, 0}>0\right)>0 & \text { for at least one } P \in \mathbf{P} .
\end{array}
$$

Lemma 17 (no arbitrage). In the financial market $\mathscr{M}$, there does not exist any arbitrage opportunity.

Proof. Assume that there exists an arbitrage opportunity; that is to say, there exist $m \leqslant 0$ and a pair $(\pi, C) \in \mathscr{A}(m)$ with $C \equiv 0$ such that $X_{T}^{m, \pi, 0} \geqslant 0$ quasi-surely for some $T>0$. Then we have $E_{G}\left(X_{T}^{m, \pi, 0}\right) \geqslant 0$. By definition of the wealth process, it has

$$
\begin{aligned}
0 & \leqslant E_{G}\left(D_{T} X_{T}^{m, \pi, 0}\right) \\
& \leqslant m+E_{G}\left(\int_{0}^{T} D_{t} X_{t}^{m, \pi, 0} \pi_{t} \nu_{t} d B_{t}\right)=m .
\end{aligned}
$$

Since the $G$-expectation of an integral with respect to $G$ Brownian motion is zero, we have $E_{G}\left(D_{T} X_{T}^{m, \pi, 0}\right)=0$. This implies $D_{T} X_{T}^{m, \pi, 0}=0$ q.s. Therefore, $(m, \pi, 0)$ cannot constitute an arbitrage.

In the financial market $\mathscr{M}$, we consider a European contingent claim $V$ and assume that its payoff at maturity time $T$ is $V_{T}$. Here, $V_{T}$ represents a nonnegative, $\mathscr{F}_{t}$-adapted random variable. Regardless of any time, we impose the assumption $V_{T} \in L_{G}^{2}\left(\Omega_{T}\right)$. The price of the claim at time 0 is denoted by $V_{0}$. For the sake of finding reasonable prices for $V$, we need to utilize the concept of arbitrage. Considering that the financial market $(\mathscr{M}, V)$ contains the original market $\mathscr{M}$ and the contingent claim $V$. Similar to the above, an arbitrage opportunity needs to be defined in the financial market $(\mathscr{M}, V)$.

Definition 18 (see [17] (arbitrage in $(\mathscr{M}, V))$ ). We say that there is an arbitrage opportunity in $(\mathscr{M}, V)$ if there exist an initial wealth $m \geqslant 0$ ( $m \leqslant 0$, resp.), an admissible pair $(\pi, C) \in \mathscr{A}(m)$, and a constant $a=-1(a=1$, resp. $)$, such that

$$
m+a \cdot V_{0} \leqslant 0
$$

at time 0 , and

$$
\begin{array}{cl}
X_{T}^{m, \pi, C}+a \cdot V_{T} \geqslant 0 & \text { q.s., } \\
P\left(X_{T}^{m, \pi, C}+a \cdot V_{T}>0\right)>0 & \text { for at least one } P \in \mathbf{P}
\end{array}
$$

at time $T$.

The values $a= \pm 1$ in Definition 18 indicate short or long positions in the claims $V$, respectively. This definition of arbitrage is standard in the literature [17]. For the same reasons as before, we again require quasi-sure dominance for the wealth at time $T$ and again with positive probability for only one possible scenario.

In the following, we show that there exist no-arbitrage prices for a claim $V$. Under these prices, there is noarbitrage opportunity. Because the uncertainty caused by the quadratic variation cannot be dispelled, generally speaking, there is no self-financing portfolio strategy which replicates the European claim or a risk-free hedge for the claim in our ambiguous market $\mathscr{M}$.

Roughly stated, since there is only one kind of situation where stocks will be traded, the measures induced by the $G$ framework result in market's incompleteness.

Definition 19 (see [17]). Given a European contingent claim $V$, the upper hedging class is defined by

$$
\mathcal{U}:=\left\{m \geqslant 0 \mid \exists(\pi, C) \in \mathscr{A}(m): X_{T}^{m, \pi, C} \geqslant V_{T} \text { q.s. }\right\}
$$

and the lower hedging class is defined by

$$
\begin{aligned}
\mathscr{L} & :=\left\{m \geqslant 0 \mid \exists(\pi, C) \in \mathscr{A}(-m): X_{T}^{-m, \pi, C}\right. \\
& \left.\geqslant-V_{T} \text { q.s. }\right\} .
\end{aligned}
$$

In addition, the upper arbitrage price is defined by

$$
v_{\text {up }}:=\inf \{m \mid m \in \mathcal{U}\}
$$

and the lower arbitrage price is defined by

$$
v_{\text {low }}:=\sup \{m \mid m \in \mathscr{L}\}
$$

Lemma 20 (see [17]). $m_{1} \in \mathscr{L}$ and $0 \leqslant n_{1} \leqslant m_{1}$ implies $n_{1} \in \mathscr{L}$. Analogously, $m_{2} \in \mathscr{U}$ and $n_{2} \geqslant m_{2}$ implies $n_{2} \in \mathscr{U}$.

The proof uses the idea that one "just consumes immediately the difference between the two initial wealth" (see [17] for the complete proof process).

For any $\sigma \in[\underline{\sigma}, \bar{\sigma}]$, we define the Black-Scholes price of a European contingent claim $V$ as follows:

$$
u_{0}^{\sigma}=E^{P^{\sigma}}\left(D_{T} V_{T}\right)
$$

Similar to the constrained circumstances [17], we prove the next three lemmas which are related to the European contingent claim $V$.

Lemma 21. For any $\sigma \in[\underline{\sigma}, \bar{\sigma}]$, it holds that $u_{0}^{\sigma}$ belongs to the interval $\left[v_{l o w}, v_{u p}\right]$.

Proof. Let $m \in \mathcal{U}$. From the definition of $\mathcal{U}$, we know that there exists a pair $(\pi, C) \in \mathscr{A}(m)$ such that $X_{T}^{m, \pi, C} \geqslant V_{T}$ 
q.s. Employing the properties of $G$-expectation as stated in Definition 1 , we obtain for any $\sigma \in[\underline{\sigma}, \bar{\sigma}]$ that

$$
\begin{aligned}
m & =E_{G}\left(m+\int_{0}^{T} D_{t} X_{t}^{m, \pi, C} \pi_{t} \nu_{t} d B_{t}\right) \\
& \geqslant E_{G}\left(m+\int_{0}^{T} D_{t} X_{t}^{m, \pi, C} \pi_{t} \nu_{t} d B_{t}-\int_{0}^{T} D_{t} d C_{t}\right) \\
& =E_{G}\left(D_{T} X_{T}^{m, \pi, C}\right) \geqslant E_{G}\left(D_{T} V_{T}\right)=\sup _{P \in \mathbf{P}} \mathbb{E}^{P}\left(D_{T} V_{T}\right) \\
& \geqslant u_{0}^{\sigma} .
\end{aligned}
$$

Therefore, $u_{0}^{\sigma} \leqslant m$. We know $v_{\text {up }}:=\inf \{m \mid m \in \mathcal{U}\}$; hence, $u_{0}^{\sigma} \leqslant v_{\text {up }}$.

Analogously, let $m \in \mathscr{L}$. By definition of $\mathscr{L}$, there exists a pair $(\pi, C) \in \mathscr{A}(-m)$ such that $X_{T}^{-m, \pi, C} \geqslant-V_{T}$ q.s. For the same reason, we obtain for any $\sigma \in[\underline{\sigma}, \bar{\sigma}]$ that

$$
\begin{aligned}
-m & =E_{G}\left(-m+\int_{0}^{T} D_{t} X_{t}^{-m, \pi, C} \pi_{t} \nu_{t} d B_{t}\right) \\
& \geqslant E_{G}\left(-m+\int_{0}^{T} D_{t} X_{t}^{-m, \pi, C} \pi_{t} \nu_{t} d B_{t}-\int_{0}^{T} D_{t} d C_{t}\right) \\
& =E_{G}\left(D_{T} X_{T}^{-m, \pi, C}\right) \geqslant E_{G}\left(-D_{T} V_{T}\right) \\
& \geqslant-E^{P^{\sigma}}\left(-D_{T} V_{T}\right)=-u_{0}^{\sigma} .
\end{aligned}
$$

Therefore, $m \leqslant u_{0}^{\sigma}$. We know $v_{\text {low }}:=\sup \{m \mid m \in \mathscr{L}\}$; hence, $v_{\text {low }} \leqslant u_{0}^{\sigma}$.

Lemma 22. For any price $V_{0}>v_{u p}$, there exists an arbitrage opportunity. Also for any price $V_{0}<v_{\text {low }}$, there exists an arbitrage opportunity.

Proof. The idea of proving this lemma comes from [8]. We only consider the case $V_{0}<v_{\text {low }}$ since the argument is similar. Assume $V_{0}<v_{\text {low }}, m \leqslant 0$ and let $-m \in\left(V_{0}, v_{\text {low }}\right)$. By definition of $v_{\text {low }}$ and Lemma 20, we deduce that $-m \in \mathscr{L}$. Hence, there exists a pair $(\pi, C) \in \mathscr{A}(-m)$ with

$$
\begin{aligned}
& X_{T}^{-m, \pi, C} \geqslant-V_{T} \quad \text { q.s., } \\
& -m-V_{0}>0 .
\end{aligned}
$$

This implies the existence of arbitrage in the sense of Definition 18. If $\exists 0<a<1$ with $-a m=V_{0}$, then $(\pi, a C) \in$ $\mathscr{A}(-a m)$ and $X_{T}^{-a m, \pi, a C}=a X_{T}^{-m, \pi, C}$. Let $P \in \mathbf{P}$; without loss of generality, we may assume $P\left(V_{T}>0\right)>0$. Due to

$$
\begin{aligned}
1 & =P\left(X_{T}^{-m, \pi, C} \geqslant-V_{T}\right) \\
& \leqslant P\left(X_{T}^{-m, \pi, C}=-V_{T}\right)+P\left(a X_{T}^{-m, \pi, C}>-V_{T}\right) \leqslant 1,
\end{aligned}
$$

we deduce

$$
P\left(X_{T}^{-m, \pi, C}=-V_{T}\right)+P\left(a X_{T}^{-m, \pi, C}>-V_{T}\right)=1 .
$$

Assume $P\left(X_{T}^{-m, \pi, C}=-V_{T}\right)=1$ and we deduce $X_{T}^{-m, \pi, C}=$ $-V_{T}$, q.s. This contradicts $X_{T}^{-m, \pi, C} \geqslant-V_{T}$ q.s. Hence,
$P\left(X_{T}^{-a m, \pi, a C}>-V_{T}\right)>0$. Hence, $(-a m, \pi, a C)$ constitutes an arbitrage.

Lemma 23. For any $V_{0} \nsubseteq \mathscr{L} \cup \mathcal{U}$, there is no arbitrage in the financial market $(\mathscr{M}, V)$.

Proof. The idea of proving this lemma also comes from [8]. We prove it by contradiction. Assume $V_{0} \nsubseteq \mathscr{U}, V_{0} \nsubseteq \mathscr{L}$ and that there exists an arbitrage opportunity in $(\mathscr{M}, V)$. We suppose that it satisfies Definition 18 for $a=1$. The case $a=-1$ works similarly.

By definition of arbitrage, there exists $m \leqslant 0,(\pi, C) \mathscr{A} \in$ $(-m)$ with

$$
\begin{aligned}
m & =X_{0}^{-m, \pi, C} \leqslant-V_{0}, \\
X_{T}^{-m, \pi, C} & \geqslant-V_{T} \quad \text { q.s. }
\end{aligned}
$$

Therefore, $m \in \mathscr{L}$. By Lemma 21, it has $V_{0} \in \mathscr{L}$. This contradicts our assumption.

Theorem 24. For the financial market $(\mathscr{M}, V)$, the following identities hold:

$$
\begin{gathered}
v_{\text {up }}=E_{G}\left(D_{T} V_{T}\right), \\
v_{\text {low }}=-E_{G}\left(-D_{T} V_{T}\right) .
\end{gathered}
$$

Proof. Firstly, let us begin with the identity $v_{\text {up }}=E_{G}\left(D_{T} V_{T}\right)$. As seen in the proof of Lemma 22, for any $m \in \mathcal{U}$ we have $m \geqslant$ $E_{G}\left(D_{T} V_{T}\right)$. Therefore, $v_{\text {up }}=\inf \{m \mid m \in \mathcal{U}\} \geqslant E_{G}\left(D_{T} V_{T}\right)$.

To show the opposite inequality we need to define the $G$ martingale $M$ by

$$
M_{t}:=E_{G}\left(D_{T} V_{T} \mid \mathscr{F}_{t}\right), \quad \forall t \leqslant T .
$$

By the martingale representation theorem [15] (see Theorem 9), we know there exists $z \in H_{G}^{1}(0, T)$ and continuous, increasing processes $K=\left(K_{t}\right)$ with $K_{T} \in L_{G}^{1}\left(\Omega_{T}\right)$ such that for any $t \leqslant T$

$$
M_{t}=E_{G}\left(D_{T} V_{T}\right)+\int_{0}^{t} z_{s} d B_{s}-K_{t} \quad \text { q.s. }
$$

For any $t \leqslant T$, we set $m=E_{G}\left(D_{T} V_{T}\right) \geqslant 0, X_{t} \pi_{t} \nu_{t}=$ $z_{t} D_{t}^{-1} \in H_{G}^{1}(0, T)$, and $C_{t}=\int_{0}^{t} D_{s}^{-1} d K_{s} \in L_{G}^{1}\left(\Omega_{T}\right)$. Then the wealth process $X^{m, \pi, C}$ satisfies

$$
\begin{aligned}
D_{t} X_{t}^{m, \pi, C} & =m+\int_{0}^{t} D_{s} X_{s}^{m, \pi, C} \pi_{s} \nu_{s} d B_{s}-\int_{0}^{t} D_{s} d C_{s} \\
& =M_{t}
\end{aligned}
$$

The properties of $K$ and $C$ obey the conditions of a cumulative consumption process in the sense of Definition 11. Due to $D_{t} X_{t}^{m, \pi, C}=M_{t} \geqslant 0$, for $\forall t \leqslant T$, the wealth process is bounded from below, where $(\pi, C)$ is admissible for $m$.

As $X_{T}^{m, \pi, C}=D_{T}^{-1} M_{T}=V_{T}$ quasi-surely, we have $m=$ $E_{G}\left(D_{T} V_{T}\right) \in \mathcal{U}$. Due to the definition of $\mathcal{U}$, we conclude that $v_{\text {up }} \leqslant E_{G}\left(D_{T} V_{T}\right)$. 
The proof for the second identity is analogous. Again, using the proof of Lemma 22, we obtain $m \leqslant-E_{G}\left(-D_{T} V_{T}\right)$ for any $m \in \mathscr{L}$. Hence, $v_{\text {low }} \leqslant-E_{G}\left(-D_{T} V_{T}\right)$.

In order to obtain $v_{\text {low }} \geqslant-E_{G}\left(-D_{T} V_{T}\right)$, we define a $G$ martingale $M$ by

$$
M_{t}=E_{G}\left(-D_{T} V_{T} \mid \mathscr{F}_{t}\right), \quad \forall t \leqslant T .
$$

The remaining part is almost a copy of the above. By the martingale representation theorem [15], there exist $z \in$ $H_{G}^{1}(0, T)$, and a continuous, increasing process $K=\left(K_{t}\right)$ with $K_{T} \in L_{G}^{1}\left(\Omega_{T}\right)$ such that, for any $t \leqslant T$,

$$
M_{t}=E_{G}\left(-D_{T} V_{T}\right)+\int_{0}^{t} z_{s} d B_{s}-K_{t} \quad \text { q.s. }
$$

As the above, for any $t \leqslant T$, let

$$
\begin{gathered}
-m=E_{G}\left(-D_{T} V_{T}\right) \geqslant 0, \\
X_{t} \pi_{t} \nu_{t}=z_{t} D_{t}^{-1} \in H_{G}^{1}(0, T),
\end{gathered}
$$

and $C_{t}=\int_{0}^{t} D_{s}^{-1} d K_{s} \in L_{G}^{1}\left(\Omega_{T}\right)$. Then the wealth process $X^{-m, \pi, C}$ satisfies

$$
\begin{aligned}
D_{t} X_{t}^{-m, \pi, C} & =-m+\int_{0}^{t} D_{s} X_{s}^{-m, \pi, C} \pi_{s} v_{s} d B_{s}-\int_{0}^{t} D_{s} d C_{s} \\
& =M_{t}
\end{aligned}
$$

where $C$ obeys the condition of a cumulative consumption process due to the properties of $K$. Moreover, for any $t \leqslant T$, it has

$$
D_{t} X_{t}^{-m, \pi, C}=E_{G}\left(-D_{T} V_{T} \mid \mathscr{F}_{t}\right) \geqslant E_{G}\left(-V_{T} \mid \mathscr{F}_{t}\right)
$$

which is bounded from below in the sense of item (iii) in Definition 15 because $-V_{T} \in L_{G}^{2}\left(\Omega_{T}\right)$. Therefore, the wealth process is bounded from below. Consequently, $(\pi, C)$ is admissible for $-m$.

Since $X_{T}^{-m, \pi, C}=D_{T}^{-1} M_{T}=-V_{T}$ q.s., it has $m=$ $-E_{G}\left(-D_{T} V_{T}\right) \in \mathscr{L}$.

Due to the definition of $\mathscr{L}$, we conclude $v_{\text {low }} \geqslant$ $-E_{G}\left(-D_{T} V_{T}\right)$. So far, we have completed the proof of Theorem 24 .

The proof of Theorem 24 is different from that of Vorbrink [8], because the volatility of stock price is related to $t$ rather than a constant.

Remark 25. Because of sublinear expectation $E_{G}$, by Theorem 24 we have $v_{\text {low }} \neq v_{\text {up }}$. This means that the market is not complete implying that not all claims can be hedged perfectly. Therefore, there are many no-arbitrage prices for $V$. As long as $\left(E_{G}\left[D_{T} V_{T} \mid \mathscr{F}_{t}\right]\right)$ is not a symmetric $G^{-}$ martingale, it has $v_{\text {low }} \neq v_{\text {up }}$. Under other circumstances, the process $K$ is identically equal to zero (see Remark 8), meaning that $\left(E_{G}\left[D_{T} V_{T} \mid \mathscr{F}_{t}\right]\right)$ is symmetric and $V_{T}$ can be hedged perfectly owing to Remark 8 and Theorem 9.
Theorem 26. For any price $V_{0} \in\left(v_{\text {low }}, v_{u p}\right) \neq \emptyset$ of a European contingent claim at time zero, there does not exist any arbitrage opportunity in $(\mathscr{M}, V)$. For any price $V_{0} \nsubseteq\left(v_{\text {low }}, v_{\text {up }}\right) \neq \emptyset$ there exists an arbitrage in the market.

Proof. The first part directly follows from Lemma 23. From Lemma 22, we know that $V_{0} \nsubseteq\left[v_{\text {low }}, v_{\text {up }}\right]$ implies the existence of an arbitrage opportunity. Thus, we only need to show that $V_{0}=v_{\text {up }}$ and $V_{0}=v_{\text {low }}$ admit an arbitrage opportunity.

We only treat the case $V_{0}=v_{\text {up }}=\inf \{m \mid m \in \mathscr{U}\}$. Then $m \geqslant V_{0}$; that is, $-m+V_{0} \leqslant 0$. The second case is similar. Comparing the proof of Theorem 24 and letting $m>0$, for $-m=E_{G}\left(-D_{T} V_{T}\right)$, there exists a pair $(\pi, C) \in \mathscr{A}(-m)$ such that

$$
\begin{aligned}
D_{t} X_{t}^{-m, \pi, C} & =-m+\int_{0}^{t} D_{s} X_{s}^{-m, \pi, C} \pi_{s} \nu_{s} d B_{s}-\int_{0}^{t} D_{s} d C_{s} \\
& =M_{t}=-D_{T} V_{T} \quad \text { q.s. }
\end{aligned}
$$

Then $K_{T}=\int_{0}^{t} D_{s} d C_{s}$, where $K$ is an increasing, continuous process with $E_{G}\left(-K_{T}\right)=0$. So we can select $P \in \mathbf{P}$ such that $E_{P}\left(-K_{T}\right)<0$ (see Remark 25). Then the pair $(\pi, C) \in \mathscr{A}(-m)$ satisfies

$$
E^{P}\left(D_{T} X_{T}^{-m, \pi, 0}\right)>E^{P}\left(D_{T} X_{T}^{-m, \pi, C}\right)=E^{P}\left(-D_{T} V_{T}\right) .
$$

Thus, $P\left(X_{T}^{m, \pi, 0}>-V_{T}\right)>0$ and we conclude that $(\pi, C) \in$ $\mathscr{A}(-m)$ constitutes an arbitrage.

On account of Theorem 26, we call $\left(v_{\text {low }}, v_{\text {up }}\right) \neq \emptyset$ the arbitrage free interval. Particularly, in the Markovian case where $V_{T}=\Phi\left(S_{T}\right)$ for some Lipschitz function $\Phi: \mathbb{R} \rightarrow \mathbb{R}$, we can give more structural details about the bounds $v_{\text {up }}$ and $v_{\text {low. }}$. We investigate this issue in Section 4.

\section{The Markovian Case}

For the European contingent claims $V$, we have the form $V_{T}=$ $\Phi\left(S_{T}\right)$ for some Lipschitz function $\Phi: \mathbb{R} \rightarrow \mathbb{R}$. We use a nonlinear Feynman-Kac formula which is established in Peng [9]. Let us rewrite the dynamics of $S$ in (22) as

$$
\begin{aligned}
& d S_{u}^{t, x}=r S_{u}^{t, x} d u+v_{u} S_{u}^{t, x} d B_{u}, \\
& u \in[t, T], S_{t}^{t, x}=x>0 .
\end{aligned}
$$

Analogy to the lower and upper arbitrage prices at time 0 , at time $t \in[0, T]$, the lower and upper arbitrage prices are noted by $v_{\text {low }}^{t}(x)$ and $v_{\text {up }}^{t}(x)$, respectively. At a considered time $t$, the stock price $S_{t}$ is replaced by the variable $x$. That is, $S_{t}=x$.

Theorem 27. Given a European contingent claim $V=\Phi\left(S_{T}\right)$, its upper arbitrage price $v_{u p}^{t}(x)$ is given by $u(t, x)$, where $u$ : $[0, T] \times \mathbb{R}_{+} \rightarrow \mathbb{R}$ is the unique solution of the following PDE:

$$
\partial_{t} u+r x \partial_{x} u+G\left(v^{2} x^{2} \partial_{x x} u\right)=r u,
$$

$$
u(T, x)=\Phi(x) \text {. }
$$


A precise representation for the corresponding trading strategy in the stock and the cumulative consumption process is given by

$$
\begin{aligned}
\beta_{t}= & \partial_{x} u\left(t, S_{t}\right), \quad \forall t \in[0, T], \\
C_{t}= & -\frac{1}{2} \int_{0}^{t} v_{s}^{2} S_{s}^{2} \partial_{x x} u\left(s, S_{s}\right) d\langle B\rangle_{s} \\
& +\int_{0}^{t} v_{s}^{2} S_{s}^{2} G\left(\partial_{x x} u\left(s, S_{s}\right)\right) d s, \quad \forall t \in[0, T] .
\end{aligned}
$$

Analogously, $-\underline{u}(t, x)$ is the lower arbitrage price $v_{\text {low }}^{t}(x)$, where $\underline{u}:[0, T] \times \mathbb{R}_{+} \rightarrow \mathbb{R}$. $v_{\text {low }}^{t}(x)$ solves (55) but with terminal condition $\underline{u}(t, x)=-\Phi(x), \forall x \in \mathbb{R}_{+}$.

Proof. Firstly, we consider the Backward Stochastic Differential Equation (in short BSDE):

$$
\begin{array}{r}
Y_{s}^{t, x}=E_{G}\left(\Phi\left(S_{T}^{t, x}\right)+\int_{s}^{T} f\left(S_{r}^{t, x}, Y_{r}^{t, x}\right) d r \mid \mathscr{F}_{s}\right), \\
s \in[t, T],
\end{array}
$$

where $f: \mathbb{R} \times \mathbb{R} \rightarrow \mathbb{R}$ is a given Lipschitz function. Peng [9] showed that the BSDE has a unique solution. So we can define a function $u:[0, T] \times \mathbb{R}_{+} \rightarrow \mathbb{R}$ by $u(t, x):=$ $Y_{t}^{t, x},(t, x) \in[0, T] \times \mathbb{R}_{+}$. In the light of the knowledge of the nonlinear Feynman-Kac formula [9], the function $u$ is a viscosity solution of the following PDE:

$$
\begin{aligned}
\partial_{t} u+r x \partial_{x} u+G\left(v^{2} x^{2} \partial_{x x} u\right)+f(x, u) & =0, \\
& u(T, x)=\Phi(x) .
\end{aligned}
$$

We define the function

$$
\widehat{u}(t, x):=E_{G}\left(\Phi\left(S_{T}^{t, x}\right) D_{T}\right) .
$$

According to the above definition, for $f \equiv 0, \widehat{u}$ solves (58). Since the function $G$ is nondegenerate, $\widehat{u}$ turns into a classical $C^{1,2}$-solution (see page 19 in [9]). Consequently, together with Itô's formula (Theorem 5.4 in [18]), it has

$$
\begin{aligned}
\widehat{u}\left(t, S_{t}^{0, x}\right)-\widehat{u}(0, x)= & \int_{0}^{t}\left[\partial_{t} \widehat{u}\left(s, S_{s}^{0, x}\right)+r S_{s}^{0, x} \partial_{x} \widehat{u}\left(s, S_{s}^{0, x}\right)\right] d s+\int_{0}^{t} \nu_{s} S_{s}^{0, x} \partial_{x} \widehat{u}\left(s, S_{s}^{0, x}\right) d B_{s} \\
& +\int_{0}^{t} \frac{1}{2}\left(\nu_{s} S_{s}^{0, x}\right)^{2} \partial_{x x} \widehat{u}\left(s, S_{s}^{0, x}\right) d\langle B\rangle_{s} \\
= & \int_{0}^{t} v_{s} S_{s}^{0, x} \partial_{x} \widehat{u}\left(s, S_{s}^{0, x}\right) d B_{s}+\underbrace{\frac{1}{2} \int_{0}^{t}\left(\nu_{s} S_{s}^{0, x}\right)^{2} \partial_{x x} \widehat{u}\left(s, S_{s}^{0, x}\right) d\langle B\rangle_{s}-\int_{0}^{t}\left(\nu_{s} S_{s}^{0, x}\right)^{2} G\left(\partial_{x x} \widehat{u}\left(s, S_{s}^{0, x}\right)\right) d s .}_{-K_{t}=-\int_{0}^{t} D_{s} d C_{s}}
\end{aligned}
$$

Now, consider the function

$$
\widetilde{u}(t, x):=D_{t}^{-1} \widehat{u}(t, x), \quad \forall(t, x) \in[0, T] \times \mathbb{R}_{+} .
$$

For $t=0$, based on Theorem 24, it has

$$
\begin{aligned}
\widetilde{u}(t, x) & =\widehat{u}(t, x)=E_{G}\left(\Phi\left(S_{T}^{t, x}\right) D_{T}\right)=E_{G}\left(D_{T} V_{T}\right) \\
& =v_{\text {up }}^{t}(x), \quad \forall(t, x) \in[0, T] \times \mathbb{R}_{+} .
\end{aligned}
$$

Moreover, $\tilde{u}$ can be used as a solution of (55). In addition, the function $u$ defined by

$$
\begin{aligned}
u(t, x):=Y_{t}^{t, x}=E_{G}\left(\Phi\left(S_{T}^{t, x}\right)-\right. & \left.\int_{t}^{T} r Y_{s}^{t, x} d s \mid \mathscr{F}_{t}\right), \\
& \forall(t, x) \in[0, T] \times \mathbb{R}_{+},
\end{aligned}
$$

solves (55) owing to the nonlinear Feynman-Kac formula since $f(x, y)=-r y$. By uniqueness of the solution in (55) (see [19]; $f$ is obviously bounded in $x$ ), we have $\widetilde{u}=u$. Thus,

$$
\begin{aligned}
u(t, x)=E_{G}\left(\Phi\left(S_{T}^{t, x}\right) D_{T-t}\right)= & v_{\mathrm{up}}^{t}(x), \\
& \forall(t, x) \in[0, T] \times \mathbb{R}_{+},
\end{aligned}
$$

and it uniquely solves (55).
In combination with the proof of Theorem 24, by using its notions and Remark 12, we obtain the precise expressions for the trading strategy $\beta$ and the cumulative consumption process $C$. That is, it has $z_{t}=v_{t} S_{t}^{0, x} \partial_{x} \widehat{u}\left(t, S_{t}^{0, x}\right)=S_{t}^{0, x} \beta_{t} v_{t} D_{t}$. Therefore, $\beta_{t}=\partial_{x} u\left(t, S_{t}\right), \forall t \in[0, T]$.

Analogously, we derive

$$
\begin{aligned}
C_{t}= & \int_{0}^{t} D_{t}^{-1} d K_{s} \\
= & -\frac{1}{2} \int_{0}^{t} v_{s}^{2} S_{s}^{2} \partial_{x x} u\left(s, S_{s}\right) d\langle B\rangle_{s} \\
& +\int_{0}^{t} v_{s}^{2} S_{s}^{2} G\left(\partial_{x x} u\left(s, S_{s}\right)\right) d s, \quad \forall t \in[0, T] .
\end{aligned}
$$

Due to Theorem 27, the functions $u(t, x)=v_{\text {up }}^{t}(x)$ and $\underline{u}(t, x)=-v_{\text {low }}^{t}(x)$ can be characterized as the unique solutions of (55). Under the circumstances of $\Phi$ being a convex or concave function, respectively, (55) simplifies greatly. 
Lemma 28. (1) If $\Phi$ is concave, then $u(t, \cdot)$ is convex for any $t \leqslant T$.

(2) If $\Phi$ is concave, then $u(t, \cdot)$ is concave for any $t \leqslant T$. Similarly, if $\Phi$ is convex, then $u(t, \cdot)$ is concave for any $t \leqslant T$. If $\Phi$ is concave, then $\underline{u}(t, \cdot)$ is convex for any $t \leqslant T$.

Proof. We only need to take into account the upper arbitrage price which is determined by the function

$$
\begin{aligned}
u(t, x)=E_{G}\left(\Phi\left(S_{T}^{t, x}\right) D_{T-t}\right)= & v_{\text {up }}^{t}(x), \\
& \forall(t, x) \in[0, T] \times \mathbb{R}_{+} .
\end{aligned}
$$

First of all, let $\Phi$ be convex, $t \in[0, T]$, and $x, y \in \mathbb{R}_{+}$. For any $\theta \in[0,1]$, it has

$$
\begin{aligned}
& u(t, \theta x+(1-\theta) y)=E_{G}\left[\Phi\left(S_{T}^{t, \theta x+(1-\theta) y}\right) e^{-r(T-t)}\right] \\
& =E_{G}[\Phi((\theta x+(1-\theta) y) \\
& \left.\left.\cdot e^{-r(T-t)-(1 / 2) \int_{t}^{T} v_{u}^{2} d\langle B\rangle_{u}+\int_{t}^{T} v_{u} d B_{u}}\right) e^{-r(T-t)}\right] \\
& \leqslant E_{G}\left[\theta \Phi \left(x e^{\left.-r(T-t)-(1 / 2) \int_{t}^{T} v_{u}^{2} d\langle B\rangle_{u}+\int_{t}^{T} v_{u} d B_{u}\right)+(1}\right.\right. \\
& \left.-\theta) \Phi\left(y e^{-r(T-t)-(1 / 2) \int_{t}^{T} v_{u}^{2} d\langle B\rangle_{u}+\int_{t}^{T} v_{u} d B_{u}}\right)\right] e^{-r(T-t)} \\
& \leqslant E_{G}\left[\theta \Phi \left(x e^{\left.-r(T-t)-(1 / 2) \int_{t}^{T} v_{u}^{2} d\langle B\rangle_{u}+\int_{t}^{T} v_{u} d B_{u}\right)}\right.\right. \\
& \left.\cdot e^{-r(T-t)}\right]+E_{G}[(1-\theta) \\
& \left.\cdot \Phi\left(y e^{-r(T-t)-(1 / 2) \int_{t}^{T} v_{u}^{2} d\langle B\rangle_{u}+\int_{t}^{T} v_{u} d B_{u}}\right)\right] \\
& =\theta E_{G}\left[\Phi\left(S_{T}^{t, x}\right) e^{-r(T-t)}\right]+(1-\theta) E_{G}\left[\Phi\left(S_{T}^{t, y}\right)\right. \\
& \left.\cdot e^{-r(T-t)}\right]=\theta u(t, x)+(1-\theta) u(t, y),
\end{aligned}
$$

where we used the convexity of $\Phi$, the monotonicity of $E_{G}$, and, in the second inequality, the sublinearity of $E_{G}$. Therefore, $u(t, \cdot)$ is convex for all $t \in[0, T]$.

Secondly, let $\Phi$ be concave. For any $(t, x) \in[0, T] \times \mathbb{R}_{+}$, we define

$$
g(t, x):=E^{P}\left[\Phi\left(\widetilde{S}_{T}^{t, x}\right) e^{-r(T-t)}\right]
$$

where

$$
d \widetilde{S}_{s}^{t, x}=r \widetilde{S}_{s}^{t, x} d s+\underline{\sigma} \widetilde{S}_{s}^{t, x} d B_{s}, \quad s \in[t, T], \widetilde{S}_{t}^{t, x}=x .
$$

Since $B=\left(B_{t}\right)$ is a classical Brownian motion under $P_{0}, g$ solves the Black-Scholes PDE (7) with $\bar{\sigma}$ replaced by $\underline{\sigma}$.

Because $E^{P}$ is linear, this is straightforward to mean that $g(t, \cdot)$ is concave for any $t \in[0, T]$. Consequently, $g$ also solves (55). By uniqueness, we conclude that $g=u$. Therefore, $u(t, \cdot)$ is concave for any $t \in[0, T]$.

\section{Conclusion}

In order to analyse the financial markets with volatility uncertainty, we consider a stock price modeled by a geometric $G$-Brownian motion which features volatility uncertainty. This is all based on the structure of a G-Brownian motion. The "G-framework" is summarized by Peng [9] which gives us a useful mathematical setting. A little new arbitrage free concept is utilized to obtain the detailed results which give us an economically better understanding of financial markets under volatility uncertainty. We establish the connection of the lower and supper arbitrage prices by means of partial differential equations. The outcomes in this paper are only applied to European contingent claims. For other cases, we would extend these results to American contingent claims in our forthcoming paper.

\section{Conflicts of Interest}

The authors declare that there are no conflicts of interest regarding the publication of this paper.

\section{References}

[1] P. Beissner, "Equilibrium prices and trade under ambiguous volatility," Economic Theory, vol. 61, no. 4, pp. 1-26, 2016.

[2] I. Gilboa and D. Schmeidler, "Maxmin expected utility with nonunique prior," Journal of Mathematical Economics, vol. 18, no. 2, pp. 141-153, 1989.

[3] P. Artzner, F. Delbaen, J.-M. Eber, and D. Heath, "Coherent measures of risk," Mathematical Finance, vol. 9, no. 3, pp. 203228, 1999.

[4] F. Maccheroni, M. Marinacci, and A. Rustichini, "Dynamic variational preferences," Journal of Economic Theory, vol. 128, no. 1, pp. 4-44, 2006.

[5] Z. Chen and L. Epstein, "Ambiguity, risk, and asset returns in continuous time," Econometrica, vol. 70, no. 4, pp. 1403-1443, 2002.

[6] D. Fernholz and I. Karatzas, "Optimal arbitrage under model uncertainty," Annals of Applied Probability, vol. 21, no. 2011, pp. 2053-2428, 2011.

[7] L. G. Epstein and S. Ji, "Ambiguous volatility and asset pricing in continuous time," Review of Financial Studies, vol. 26, no. 7, pp. 1740-1786, 2013.

[8] J. Vorbrink, "Financial markets with volatility uncertainty," Journal of Mathematical Economics, vol. 53, no. 8, pp. 64-78, 2014.

[9] S. Peng, "Nonlinear expectations and stochastic calculus under uncertainty," 2010, https://arxiv.org/abs/1002.4546.

[10] S. Peng, "G-Brownian motion and dynamic risk measure under volatility uncertainty," 2007, https://arxiv.org/abs/0711.2834.

[11] L. Denis, M. Hu, and S. Peng, "Function spaces and capacity related to a sublinear expectation: application to G-Brownian motion paths," Potential Analysis, vol. 34, no. 2, pp. 139-161, 2011.

[12] L. Denis and C. Martini, "A theoretical framework for the pricing of contingent claims in the presence of model uncertainty," The Annals of Applied Probability, vol. 16, no. 2, pp. 827-852, 2006. 
[13] M. Avellaneda, A. Levy, and A. Paras, "Pricing and hedging derivative securities in markets with uncertain volatilities," Applied Mathematical Finance, vol. 2, no. 2, pp. 73-88, 1995.

[14] Y. Song, "Properties of hitting times for $G$-martingales and their applications," Stochastic Processes and Their Applications, vol. 121, no. 8, pp. 1770-1784, 2011.

[15] Y. Song, "Some properties on G-martingale decomposition," Science China Mathematics, vol. 54, no. 2, pp. 287-300, 2011.

[16] D. Duffie, Dynamic Asset Pricing Theory, Princeton University Press, New Jersey, NJ, USA, 1992.

[17] I. Karatzas and S. G. Kou, "On the pricing of contingent claims under constraints," The Annals of Applied Probability, vol. 6, no. 2, pp. 321-369, 1996.

[18] X. Li and S. Peng, "Stopping times and related Itô's calculus with G-Brownian motion," Stochastic Processes and Their Applications, vol. 121, no. 7, pp. 1492-1508, 2011.

[19] H. Ishii and P.-L. Lions, "Viscosity solutions of fully nonlinear second-order elliptic partial differential equations," Journal of Differential Equations, vol. 83, no. 1, pp. 26-78, 1990. 


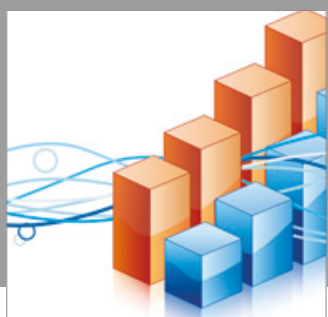

Advances in

Operations Research

vatersals

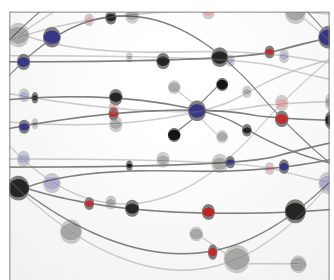

\section{The Scientific} World Journal
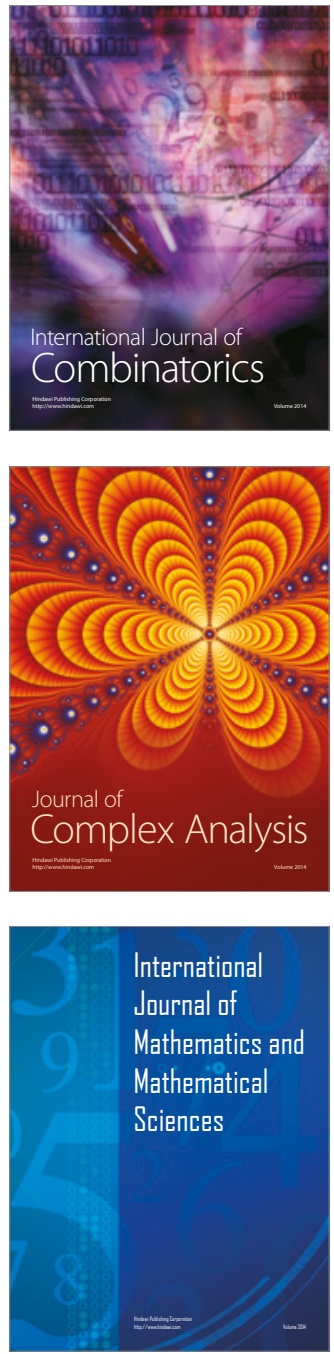
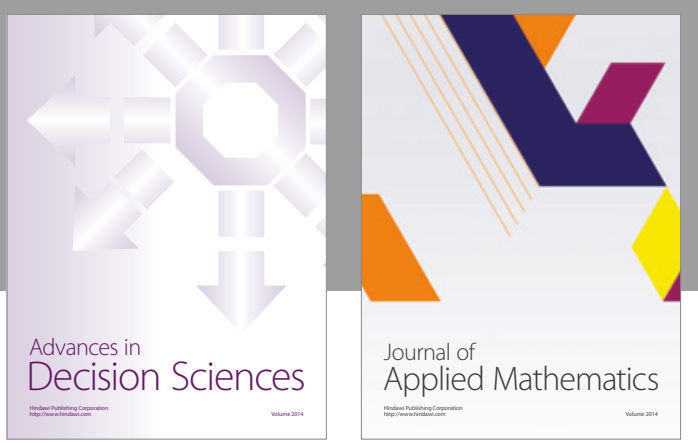

Algebra

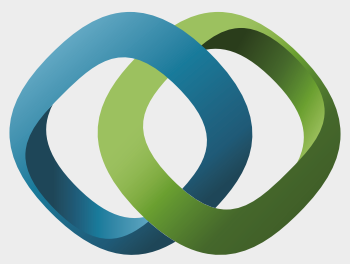

\section{Hindawi}

Submit your manuscripts at

https://www.hindawi.com
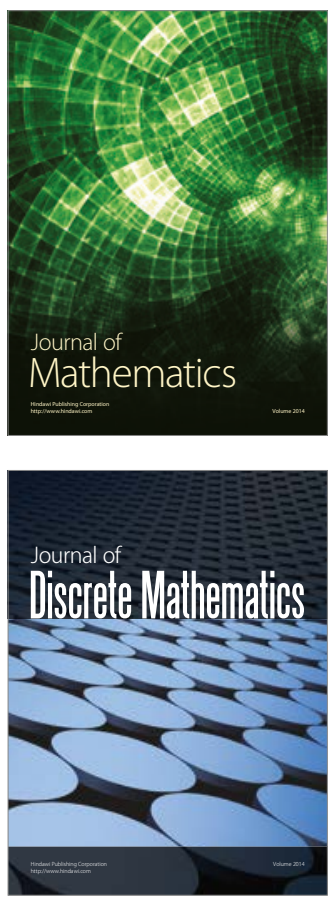

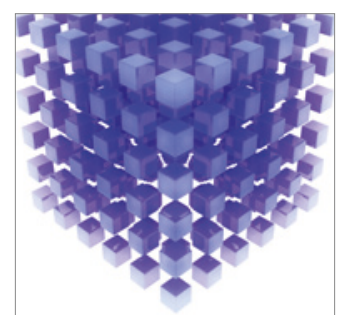

Mathematical Problems in Engineering
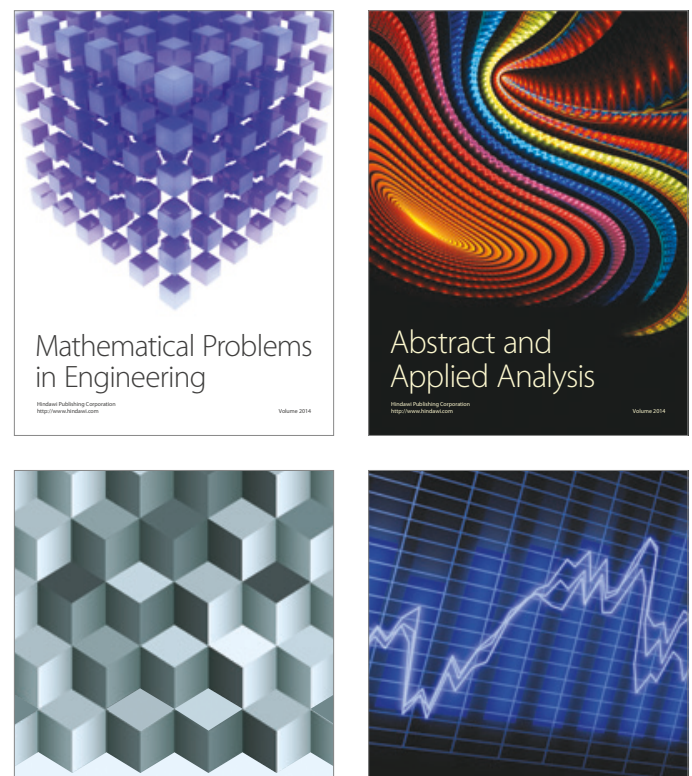

Journal of

Function Spaces

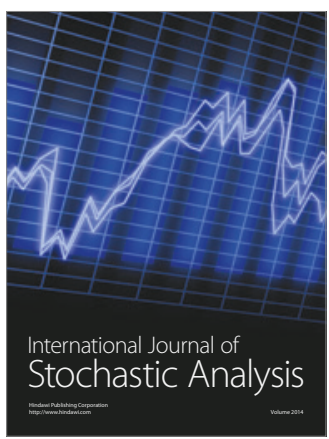

Probability and Statistics
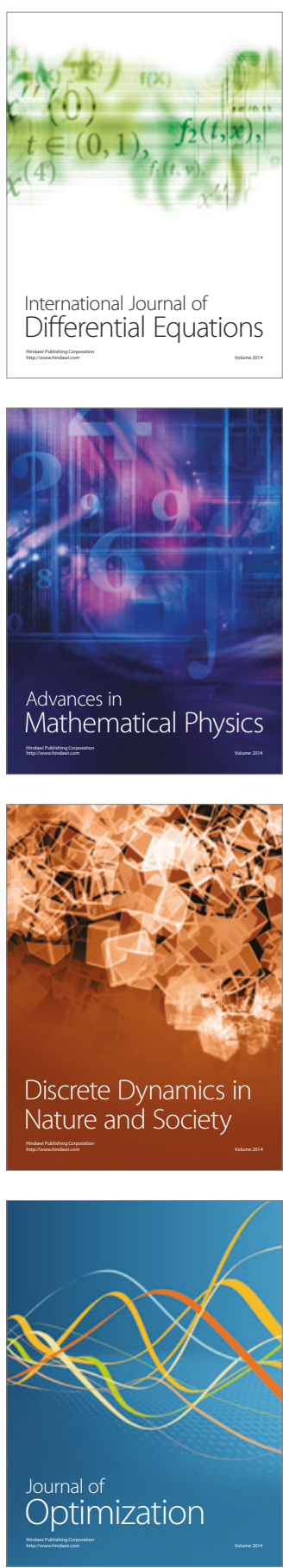